\title{
Análisis de la estabilidad y adaptabilidad de caracteres de interés agronómico en genotipos selectos de cebadilla criolla (Bromus catharticus)
}

\author{
Abbott, L. y S. Pistorale
}

\begin{abstract}
RESUMEN
Trece genotipos de Bromus catharticus se evaluaron, durante 3 años, utilizando un diseño experimental completamente aleatorizado con seis repeticiones. Por planta individual se analizaron el número de panojas, el número de semillas y el peso de 1000 semillas. La interacción genotipo x ambiente fue estadísticamente significativa e indica un comportamiento diferencial de los genotipos evaluados a través de los años. Una vez comprobada la interacción, se aplicaron tres metodologías para evaluar la estabilidad y adaptabilidad de los genotipos: la ecovalencia de Wricke, el índice de Lin \& Binns y el modelo de Eberhart \& Russell. Estas dos últimas metodologías coinciden en identificar como estables y adaptables a los mismos genotipos para los caracteres estudiados, mientras que la coincidencia con Wricke fue parcial. Considerando las tres metodologías usadas, la de Lin \& Binns es fácil de aplicar e interpretar, ya que siempre asocia mayor productividad con mayor estabilidad, y no presenta las restricciones de uso de la regresión. Sin embargo, es necesario acumular más resultados antes de proceder a la generalización de su uso.
\end{abstract}

Palabras clave: cebadilla criolla, interacción genotipo $x$ ambiente, rendimiento en semilla, mejoramiento genético.

Abbott, L. and S. Pistorale. 2011. Stability and adaptability analysis of characters of agronomical interest in selected genotypes of prairie grass (Bromus catharticus). Agriscientia XXVIII (2): 109-117

\section{SUMMARY}

Thirteen genotypes of Bromus catharticus were evaluated over a three year period, using a completely randomized experimental design with six replications. Individual plants were analyzed for the number of panicles, number of seeds and weight of 1000 seeds. The genotype $x$ environment interaction was statistically significant and it indicates a different performance of genotypes throughout the years. After verifying the interaction, three methodologies were applied in order to assess the stability and adaptability of the genotypes. These were: the 
Wricke's ecovalence, the Lin \& Binns index and the model by Eberhart \& Russell. These last two methods agree in identifyinthe same genotypes for the traits studied as stable and adaptable, while the coincidence with Wricke's method was partial. Considering the three methodologies used, the index of Lin \& Binns is easy to apply and to interpret because it is always related to higher yield, greater stability, and it does not present any of the restrictions of using regressions. However, it is necessary to gather more information before this method can be widely used.

Key words: prairie grass, genotype-environment interaction, yields of seed, genetic improvement.

L. Abbott y S. Pistorale. Departamento de Ciencias Básicas, Universidad Nacional de Luján. CC 221 (6700) Luján, Buenos Aires, Argentina. Correspondencia a L. Abbott: genetica@unlu.edu.ar

\section{INTRODUCCIÓN}

La cebadilla criolla (Bromus catharticus Vahl.; syn. B. willdenowii Kunth, B. unioloides Kunth; también llamada rescue-grass o praire-grass) es uno de los pastos nativos mas cultivados en la Argentina y de las pocas especies forrajeras autóctonas que se cultivan en Bolivia, Brasil, Chile, Ecuador, Perú y Uruguay. Es una especie anual o bianual, considerada preferentemente autógama, de ciclo otoño-inverno-primaveral, con alta capacidad de implantación y buen crecimiento inicial. Se consocia muy bien con otras especies, formando pasturas de óptima calidad en las cuales permanece por muchos años a causa de su gran producción de semilla y su resiembra natural (Wolff et al., 1996).

El amplio rango de ambientes climáticos y edáficos en los que se cultiva, puede provocar respuestas diferenciales del comportamiento de los genotipos en los diferentes ambientes como resultado de la interacción genotipo por ambiente $(G \times A)$. La interacción indica que el comportamiento de los genotipos no fue consistente a través de los ambientes (Ferreira et al., 2006). Campbell \& Jones (2005) definen la interacción G x A como la respuesta diferencial de los genotipos para una determinada característica en diferentes ambientes. Borém \& Miranda (2009) señalan que las variedades cultivadas en ambientes diferentes pueden tener comportamientos diferentes.

El concepto de ambiente ha sido discutido ampliamente por gran número de investigadores. Allard (1960) lo expresa como la suma de todas las condiciones externas que afectan el crecimiento y desarrollo de un organismo. Según Lin et al (1986), el efecto ambiental sobre un genotipo depende del suelo y de las condiciones atmosféricas. Para estos investigadores el suelo permanece casi constante de año en año y puede ser, a pesar de todo, considerado como un efecto fijo. El tiempo atmosférico es más complejo, porque tiene una parte predecible representada por la zona climática general y una parte no predecible representada por la variación del clima, año en año.

En el mejoramiento de plantas, el estudio de la interacción $\mathrm{G} \times \mathrm{A}$ es esencial para la eficiencia del proceso selectivo, debido a que la mayoría de los caracteres de importancia agronómica son de herencia cuantitativa. Estos caracteres presentan distribución continúa, poseen herencia poligénica y son muy influenciados por las variaciones del ambiente (Chaves, 2001).

Cuando la interacción genotipo $x$ ambiente es significativa a través de cada ambiente, se ve reducida la utilidad de los promedios de los genotipos sobre todos los ambientes para la identificación de genotipos superiores. La detección de interacción $G \times A$ en ensayos de campo ha llevado al desarrollo de procedimientos que son llamados genéricamente análisis de estabilidad. Según Cruz et al. (2004) los análisis de estabilidad y adaptabilidad, posibilitan la identificación de cultivares de comportamiento previsible y que respondan a las variaciones ambientales. La estabilidad del rendimiento se refiere a su capacidad para proporcionar un comportamiento muy previsible, incluso con los cambios ambientales (Borém, 2001).

Becker (1981) distingue dos tipos de estabilidad. Por una parte, la estabilidad biológica con un sentido homeostático, mediante el cual un genotipo mantiene un rendimiento constante en diferentes ambientes; este tipo de estabilidad no es deseable en la agricultura moderna, donde los 
genotipos deberían responder a las condiciones del medio mejoradas. El segundo concepto es el de estabilidad agronómica, el cual implica que un genotipo es considerado estable si rinde relativamente bien respecto al potencial de los ambientes evaluados, mostrando una baja interacción en términos de ecovalencia.

Léon (1986) considera dos conceptos diferentes de estabilidad. La estabilidad estática es cuando un material posee un rendimiento aceptable, sin tener en cuenta la variación de las condiciones ambientales; este material estable no muestra desviación del nivel esperado para el carácter en estudio, lo que significa que su variancia entre ambientes es cero. Por otro lado, la estabilidad dinámica es aquella que muestra la menor desviación de su respuesta a los ambientas. El concepto de estabilidad dinámica y el concepto de estabilidad agronómica son equivalentes, mientras que el concepto estático de Léon coincide con el concepto estático de Becker (Léon \& Becker, 1988).

La adaptabilidad fenotípica evalúa el comportamiento de los genotipos en localidades diferentes. Los estudios de adaptabilidad y de estabilidad fenotípica, para fines de mejoramiento, se refieren a la evaluación de la respuesta diferencial de los genotipos a la variación de las condiciones del ambiente. La evaluación de los cultivares se debe realizar en localidades representativas de la región y en varios años, para que se tenga seguridad en una recomendación (Fan et al., 2007).

En general, se ha aceptado que a mayor variabilidad genética de una especie, mayor su estabilidad sobre el ambiente. Allard \& Bradshaw (1964) indican que una variedad puede estar compuesta por un número de individuos diferentes, cada uno adaptado a un rango diferente de ambientes (homeostasis poblacional), o puede estar conformado por individuos semejantes, pero cada uno adaptado a un rango de ambientes (homeostasis individual). Pandey y Vargas (1985) indican que para el caso de las especies autógamas, en donde las poblaciones son de genotipo homocigótico, cada planta esta adaptada a un grupo de condiciones ambientales y tiene homeostasis individual. Por otra parte, cuando un cultivar es una mezcla de genotipos, los diferentes genotipos pueden adaptarse a diferentes condiciones ambientales con el resultado de que el cultivar tenga mayor adaptación. Este mecanismo de estabilidad se debe a la homeostasis poblacional y se atribuye a la heterogeneidad del cultivar.
En los programas de mejoramiento, tanto en la fase de selección como en la de recomendación de los genotipos, la interacción genotipo x ambiente es un gran problema para los mejoradores; por ello se realizan pruebas de estabilidad y de adaptación, mediante las cuales se hace posible identificar genotipos de interés. La elección del método para la caracterización de los genotipos en cuanto a estabilidad y adaptabilidad depende de los datos experimentales disponibles, de la precisión requerida y del tipo de información deseada por el mejorador (Cruz et al., 2004). Es importante que se utilice más de un método pues cada uno presenta peculiaridades que pueden contribuir a mejorar el análisis y, en algunos casos, los métodos pueden ser complementarios entre sí (Pereira et al., 2009).

Entre las diversas técnicas disponibles para los estudios de la estabilidad y adaptabilidad fenotípica se han utilizado la ecovalencia de Wricke, el índice de Lin \& Binns y el método de Eberhart \& Russell, porque son de simple ejecución, fácil interpretación y pueden ser aplicados cuando el número de ambientes es reducido. Se descartaron los métodos multivariados y de ordenación porque, en general, requieren de un alto número de genotipos, de un gran número localidades y son de mayor utilidad cuando el fin es dar una recomendación regionalizada (Gauch, 1990; Gauch \& Zobel, 1996).

El objetivo del presente trabajo fue evaluar y comparar diferentes métodos de análisis de la estabilidad y la adaptabilidad fenotípica, en 13 genotipos de cebadilla criolla, y determinar el método que permita identificar de forma práctica y simple los mejores genotipos.

\section{MATERIALES Y MÉTODOS}

\section{Materiales}

Se seleccionaron 13 genotipos de cebadilla criolla de localidades diferentes (Wolff et al., 2006). Por planta individual se evaluaron los siguientes caracteres: número de panojas, número de semillas llenas y peso de 1000 semillas (g).

\section{Diseño, análisis estadísticos y genéticos}

El ensayo se condujo en macetas individuales en el campo experimental de la Universidad Nacional de Luján (Buenos Aires, Argentina). Se realizó un diseño completamente aleatorizado con 13 genotipos y 6 repeticiones, donde la unidad experimental estuvo representada por 6 plantas. El manejo agronómico (fecha de siembra, trasplante, 
riego, etc.) fue el mismo durante los 3 años y lo que varió fue la condición climática de esos años, por lo que en este trabajo los ambientes corresponden a los años.

Los datos experimentales se analizaron utilizando el programa estadístico InfoStat (2008). Se realizó un análisis de varianza para detectar diferencias significativas entre genotipos, ambientes e interacción $\mathrm{G} \times \mathrm{A}$, considerando efecto fijo a los genotipos y aleatorio a los años. Para medir la interacción $\mathrm{G} \times \mathrm{A}$, los datos obtenidos en los tres años fueron analizados de manera combinada, de acuerdo al siguiente modelo estadístico:

$$
Y_{i j}=\mu+\alpha_{i}+\beta_{j}+(\alpha \beta)_{i j}+\varepsilon_{i j}
$$

donde, $Y_{i j}=$ valor medio de la variedad $i$ en el ambiente $j ; \mu=$ media general; $\alpha_{i}=$ efecto del genotipo $i$ donde $i=1-13 ; \beta_{j}=$ efecto del año $j$ (ambiente) donde $j=1-3 ;(\alpha \beta)_{i j}=$ efecto de la interacción del genotipo $i$ con el año $j ; \varepsilon_{i j}=$ efecto aleatorio o error experimental. Una vez detectada la interacción $\mathrm{G} \times \mathrm{A}$ se procedió a determinar la estabilidad y adaptabilidad de los genotipos por los tres métodos.

\section{Método de la ecovalencia}

Es el método más simple fundamentado en el concepto dinámico de estabilidad. Este método fue propuesto por Wricke (1962), quien define el término ecovalencia como la contribución de cada genotipo, en todos los ambientes, a la suma de cuadrados de la interacción $\mathrm{G} \times \mathrm{A}$. Si la ecovalencia es pequeña la estabilidad es alta.

La ecovalencia de Wricke $\left(W_{i}\right)$ fue calculada con la siguiente fórmula:

$$
W_{i}=S_{j}\left(Y_{i j}-Y_{i .}-Y_{. j}+Y_{. .}\right)^{2}
$$

donde, $W_{i}=$ valor de la ecovalencia del genotipo i; $S_{j}=$ sumatoria de los $j$ valores de ambiente en cada genotipo; $Y_{i j}=$ media del genotipo $i$ en el ambiente $j ; Y_{i .}=$ media del genotipo $i$ en los $j$ ambientes; $Y_{. j}=$ media del ambiente $j ; Y_{. .}=$media general de los ambientes.

\section{Índice de Lin \& Binns}

El Índice de Lin \& Binns (1988) constituye una medida única de la estabilidad y superioridad del comportamiento de un genotipo, y es definido como el cuadrado medio de la distancia entre la respuesta de un genotipo y el genotipo de máxima respuesta en un ambiente dado. Con este índice, la máxima respuesta en un ambiente se convierte en el testigo a considerar. El genotipo con valor de ecovalencia más bajo es el más cercano al óptimo, ya que permanecería dentro de los más productivos a través de ambientes (Scapim et al., 2000; Elias et al., 2005)

El Índice de Lin \& Binns $\left(P_{j}\right)$ fue calculado con la siguiente expresión:

$$
P_{i}=\frac{\sum_{j=1}^{a}\left(\bar{Y}_{i j}-\bar{M}_{j}\right) 2}{2 a}
$$

donde, $P_{i}=$ índice de estabilidad y superioridad del genotipo $i ; Y_{i j}=$ rendimiento medio del genotipo $i$ en el ambiente $j ; M_{j}=$ rendimiento medio máximo en al ambiente $j ; a=$ número de ambientes evaluados.

\section{Método de Eberhart \& Russell}

Eberhart \& Russell (1966) propusieron un modelo de regresión lineal para el estudio de la estabilidad y adaptabilidad fenotípica de cultivares ampliamente utilizado, en este tipo de estudios, en todo el mundo. En este modelo, además del promedio general y del coeficiente de regresión lineal de cada genotipo, es también considerado como parámetro de estabilidad la varianza de los desvíos de la regresión de cada genotipo. Este tipo de análisis fue clasificado por Becker (1981) como de estabilidad en el sentido agronómico. El efecto del ambiente puede ser descompuesto en dos componentes, uno lineal y el otro no lineal. El coeficiente de regresión está asociado con el componente lineal, indicando la adaptabilidad del genotipo, o bien, su capacidad de respuesta entre los distintos ambientes. Los desvíos de la regresión están asociados al componente no lineal e indican estabilidad genotípica. De acuerdo con este modelo, un genotipo es estable cuando presenta una media superior a la media general, un coeficiente de regresión igual a uno y desviaciones de la regresión lineal tan pequeñas como sea posible.

Eberhart \& Russell establecieron el siguiente modelo para estimar la estabilidad:

$$
Y_{i j}=\mu_{i}+\beta_{i} I_{j}+\delta_{i j}+\varepsilon_{i j}
$$

donde, $Y_{i}=$ promedio del genotipo $i$ en el ambiente $j ; \mu_{i}=$ media del genotipo $i$ en todos los ambientes; $\beta_{i}=$ coeficiente de regresión que mide la respuesta del genotipo $i$ en los diferentes ambientes; $I_{j}=$ índice ambiental; $\delta_{i j}=$ desvío de la regresión del genotipo $i$ en el ambiente $j ; \boldsymbol{\varepsilon}_{i j}$ = desviación de la regresión de la variedad y el ambiente. El índice ambiental, en cada ambiente, fue calculado por el desvío del promedio de todos los genotipos en ese ambiente, en relación con el promedio general: $I_{j}=Y_{. j}-Y_{. .}$. El coeficiente de regresión del genotipo $i$ fue estimado como: $\beta_{i}=\frac{\sum Y_{i j} I_{j}}{\sum I_{j}^{2}}$ El segundo parámetro de estabilidad, 
que tiene que ver con las desviaciones, fue estimado según la siguiente fórmula:

$$
S_{d i}^{2}=\left(\frac{\sum \delta_{i j}^{2}}{n-2}\right)-\frac{S_{e}^{2}}{r}
$$

donde, $\delta_{i j}=$ desviación del genotipo $i$ en el ambiente $i$ de regresión y es dado como: $\delta_{i j}=Y_{i j}-\hat{Y}_{i j}$, donde $\hat{Y}_{i j}$ es el valor esperado del genotipo $i$ en el ambiente $j$ y donde $S_{e}^{2} / r$ es el error residual del análisis de varianza combinado (que incluye todos los ambientes).

De acuerdo con esta metodología, la adaptabilidad corresponde a la capacidad de los genotipos de aprovechar ventajosamente los estímulos del ambiente. Valores del coeficiente $\beta_{1}>1$, indican adaptabilidad específica a ambientes favorables, pero comportamiento pobre en los ambientes desfavorables. En cambio, si es $\beta_{1}<1$, indica adaptabilidad específica a ambientes desfavorables. Si tiene valores de $\beta_{1}=0$ significa adaptabilidad general, es decir, mantiene un promedio constante en todos los ambientes; si está asociado con promedios altos, se caracterizará como genotipo ideal. Son considerados estables los genotipos con desvíos de la regresión no significativos $\left(S_{d i}^{2}=0\right)$ e inestables aquellos con desvíos estadísticamente significativos.

Se realizaron las siguientes pruebas de hipótesis para los parámetros de Eberhart \& Russell:

Coeficiente de regresión:

$$
\begin{aligned}
& \left.\mathrm{H}_{0}\right) \beta_{1}=1 \mathrm{t}_{\text {calculado }}=\frac{b_{i}-1}{\sqrt{\operatorname{Var}\left(b_{i}\right)}} \\
& \text { donde, } \operatorname{Var}\left(b_{i}\right)=S_{d i}^{2} / \sum_{j} I_{j}^{2}
\end{aligned}
$$

Desvíos de la regresión de cada genotipo:

$$
\left.H_{0}\right) S_{d i}^{2}=0 ; \quad F_{\text {calculado }}=\frac{\left(\sum_{j} \delta_{i j}^{2} / a-2\right)}{\text { Error combinado }}
$$

\section{RESULTADOS Y DISCUSIÓN}

\section{Análisis de la variabilidad}

Las pruebas multivariadas que utilizan los estadísticos de Pillai, Wilks, Lawley-Hotelling y Roy para evaluar la variabilidad de los 13 genotipos durante los 3 años mostraron diferencias estadísticamente significativas $(p<0,0001)$ para los tres caracteres estudiados en todos los genotipos evaluados.

\section{Interacción genotipo por ambiente}

La interacción genotipo por ambiente se define como la respuesta diferencial de un grupo de genotipos frente a distintos ambientes. En este estudio, los años fueron considerados como ambientes y las diferencias fueron causadas por las variaciones climáticas (temperaturas, lluvia y radiación solar incidente) en cada etapa fenológica. Los genotipos, los años y la interacción $\mathrm{G} \times \mathrm{A}$ fueron significativos para todos los caracteres estudiados, y esto significa que los genotipos evaluados se comportaron en forma diferente en los años de evaluación, así como entre ellos mismos (Tabla 1).

\section{Análisis de la estabilidad}

\section{Número de panojas}

Una vez evidenciada la presencia de interacción $\mathrm{G} \times \mathrm{A}$ fue calculada la estabilidad y la adaptabilidad por los tres métodos. El parámetro de estabilidad de Wricke $\left(W_{i}\right)$ se obtiene al fraccionar el cuadrado medio de la interacción $G \times A$ en tantos componentes como genotipos fueron evaluados. En la Tabla 2 se observan los valores de ecovalencia de Wricke. De ellos, los

\begin{tabular}{|c|c|c|c|c|c|c|c|}
\hline \multirow{2}{*}{ FV } & \multirow{2}{*}{ gl } & \multicolumn{2}{|c|}{$N^{\circ}$ de panojas } & \multicolumn{2}{|c|}{$N^{\circ}$ de semillas } & \multicolumn{2}{|c|}{ Peso 1000 semillas } \\
\hline & & $\mathrm{CM}$ & $\mathrm{p}$ & $\mathrm{CM}$ & $\mathrm{p}$ & $\mathrm{CM}$ & $p$ \\
\hline Modelo & 38 & 73,21 & 0,0001 & 6817,30 & 0,0001 & 15,51 & 0,0001 \\
\hline Genotipo (G) & 12 & 61,93 & 0,0001 & 15326,41 & 0,0001 & 27,96 & 0,0001 \\
\hline Año (A) & 2 & 689,82 & 0,0001 & 7605,61 & 0,0091 & 99,57 & 0,0001 \\
\hline$G \times A$ & 24 & 27,46 & 0,0001 & 2497,05 & 0,0486 & 2,27 & 0,0025 \\
\hline Error & 195 & 7,77 & & 1581,09 & & 1,06 & \\
\hline Total & 233 & & & & & & \\
\hline$R^{2}$ & & \multicolumn{2}{|c|}{0,58} & \multicolumn{2}{|c|}{0,35} & \multicolumn{2}{|c|}{0,69} \\
\hline CV & & \multicolumn{2}{|c|}{30,83} & \multicolumn{2}{|c|}{25,54} & \multicolumn{2}{|c|}{10,80} \\
\hline
\end{tabular}
cinco genotipos que presentaron los valores más

Tabla 1. Análisis de varianza para el número de panojas por planta, el número de semillas por planta y el peso de 1000 semillas, coeficiente de determinación $\left(R^{2}\right)$ en y coeficiente de variación ( $C V \%$ ) en cebadilla criolla en tres años de estudio. 
Tabla 2. Valores medios, media general $(\mu)$, ecovalencia de Wricke $\left(W_{i}\right)$ en porcentaje e índice de Lin \& Binns $\left(P_{i}\right)$ en porcentaje, para el número de panojas por planta, el número de semillas por planta y el peso de 1000 semillas en cebadilla criolla en tres años de estudio.

\begin{tabular}{|c|c|c|c|c|c|c|c|c|c|}
\hline \multirow[b]{2}{*}{ genotipo } & \multicolumn{3}{|c|}{$\begin{array}{c}N^{\circ} \text { de panojas } \\
\mu=9,04\end{array}$} & \multicolumn{3}{|c|}{$\begin{array}{c}N^{\circ} \text { de semillas } \\
\mu=155,69\end{array}$} & \multicolumn{3}{|c|}{$\begin{array}{c}\text { Peso } 1000 \text { semillas } \\
\mu=9,04\end{array}$} \\
\hline & Media & $W_{i}$ & $P_{i}$ & Media & $W_{i}$ & $P_{i}$ & Media & $W_{i}$ & $P_{i}$ \\
\hline 1 & 8,33 & 0,35 & 9,18 & 141,06 & 0,96 & 8,84 & 10,19 & 13,28 & 4,45 \\
\hline 2 & 8,28 & 0,46 & 7,88 & 122,13 & 0,04 & 14,24 & 12,11 & 52,31 & 0,03 \\
\hline 3 & 10,11 & 17,40 & 4,03 & 135,65 & 1,01 & 12,04 & 8,12 & 1,54 & 14,33 \\
\hline 4 & 10,72 & 3,28 & 2,21 & 124,39 & 27,72 & 16,08 & 8,56 & 2,18 & 11,89 \\
\hline 5 & 7,39 & 2,94 & 11,77 & 161,40 & 0,54 & 5,08 & 11,13 & 1,95 & 1,52 \\
\hline 6 & 7,83 & 19,35 & 12,13 & 152,49 & 2,33 & 7,13 & 8,05 & 3,61 & 15,14 \\
\hline 7 & 5,72 & 18,83 & 21,03 & 151,89 & 2,58 & 6,47 & 8,75 & 8,32 & 10,47 \\
\hline 8 & 9,00 & 0,04 & 6,18 & 202,57 & 28,84 & 0,22 & 8,77 & 0,51 & 10,32 \\
\hline 9 & 12,94 & 2,08 & 0,39 & 150,67 & 0,03 & 6,84 & 10,85 & 0,01 & 1,91 \\
\hline 10 & 8,72 & 0,31 & 6,39 & 212,07 & 23,75 & 0,00 & 8,87 & 5,70 & 10,19 \\
\hline 11 & 9,67 & 7,61 & 4,18 & 124,48 & 11,27 & 14,82 & 9,06 & 2,13 & 8,56 \\
\hline 12 & 7,83 & 1,80 & 11,95 & 156,85 & 0,90 & 6,15 & 10,06 & 7,12 & 4,68 \\
\hline 13 & 11,00 & 25,55 & 2,69 & 188,26 & 0,01 & 2,09 & 9,59 & 1,34 & 6,50 \\
\hline
\end{tabular}

bajos de ecovalencia fueron 8, 10, 1, 2 y 12; son considerados los más estables, pero todos ellos tuvieron una media inferior a la media general por lo cual no fueron seleccionados. Los genotipos 9 , y 4 presentaron una media superior a la media general y los valores de ecovalencia fueron relativamente bajos y podrían ser incorporados en un programa de selección.

El índice de Lin \& Binns $\left(P_{i}\right)$ constituye una medida única de la estabilidad y superioridad del comportamiento de un genotipo. Con este índice, los cinco genotipos con menor valor de $P_{i}$ fueron 9 , $4,13,3$ y 11 y tuvieron un rendimiento superior el promedio general (Tabla 2).

En la Tabla 3 se muestran los valores medios y los parámetros de adaptabilidad $\left(\beta_{i}\right)$ y estabilidad $\left(S_{d i}^{2}\right)$ de Eberhart \& Russell, de los 13 genotipos para el número de panojas por planta. La prueba de hipótesis para $\beta_{1} \beta_{i}=1$ resultó significativamente distinta de 1 para los genotipos 6 y 7 , por lo que se consideran no adaptables. Para el resto de los genotipos no hay evidencia suficiente para rechazarla, lo que indica adaptabilidad, general o pobre, según su media sea superior o inferior a la media general. Con respecto a $S_{d i}^{2}$, para los genotipos 9 y 12 resultó significativamente distinto de 0 lo que indica que son inestables. Según esta metodología, se podrían seleccionar los genotipos $3,4,11$, y 13 , que poseen adaptación general, son estables y su media supera a la media general, y el genotipo 8 cuya media es igual a la media general.

Tabla 3. Parámetros de adaptabilidad $\left(\beta_{i}\right)$, de estabilidad $\left(S_{d i}^{2}\right)$, media general $(\mu)$ y coeficiente de determinación $\left(R^{2}\right)$ para el número de panojas por planta en cebadilla criolla en tres años de estudio.

\begin{tabular}{|c|c|c|c|c|c|c|}
\hline Genotipo & Media & $\mu=9,04$ & $\beta_{i}$ & $S_{d i}^{2}$ & Interpretación & $R^{2}$ \\
\hline 1 & 8,33 & $<$ & 0,74 & $1,15 \mathrm{~A}$ & Ios ambientes. Estable. & 39,12 \\
\hline 2 & 8,28 & $<$ & 0,99 & $1,31 \mathrm{~A}$ & Ios ambientes. Estable. & 54,21 \\
\hline 3 & 10,11 & $>$ & 1,62 & $0,82 \mathrm{~A}$ & s los ambientes. Estable. Genotipo ideal. & 54,27 \\
\hline 4 & 10,72 & $>$ & 1,47 & $1,54 \mathrm{~A}$ & s los ambientes. Estable. Genotipo ideal. & 65,79 \\
\hline 5 & 7,39 & $<$ & 0,61 & $1,17 \mathrm{~A}$ & Ios ambientes. Estable. & 46,33 \\
\hline 6 & 7,83 & $<$ & 0,11 & $1,29 N$ & & 1,28 \\
\hline 7 & 5,72 & $<$ & 0,13 & $0,19 \mathrm{~N}$ & & 3,46 \\
\hline 8 & 9,00 & $=$ & 1,05 & $1,29 \mathrm{~A}$ & tes. Estable. & 43,22 \\
\hline 9 & 12,94 & $>$ & 1,54 & $10,67 \mathrm{~A}$ & s los ambientes. Inestable. & 64,45 \\
\hline 10 & 8,72 & $<$ & 1,23 & $0,44 \mathrm{~A}$ & Ios ambientes. Estable. & 45,30 \\
\hline 11 & 9,67 & $>$ & 1,54 & $1,11 \mathrm{~A}$ & s los ambientes. Estable. Genotipo ideal. & 56,74 \\
\hline 12 & 7,83 & $<$ & 0,49 & $5,25 \mathrm{~A}$ & los ambientes. Inestable. & 19,12 \\
\hline 13 & 11,00 & $>$ & 1,73 & $0,99 \mathrm{~A}$ & s los ambientes. Estable. Genotipo ideal. & 61,78 \\
\hline
\end{tabular}


Los valores del coeficiente de determinación $\left(R^{2}\right)$ aparecen tabulados en la última columna de la Tabla 3. La magnitud del valor de este coeficiente indica la proporción de la varianza de la variable producción de panojas que puede ser atribuida a la variación del índice ambiental. Sus valores muestran que entre un 19,12 y un $65,79 \%$ de la variación en la producción de los genotipos se debe a la respuesta lineal de éstos frente a los cambios ambientales. La excepción son los genotipos 6 y 7 con valores de $R^{2}$ de 1,28 y $3,46 \%$ respectivamente, que no poseen adaptabilidad como lo confirma la no aceptación de la hipótesis de $\beta_{1}$.

Los mejores genotipos, para el número de panojas por planta, por Lin \& Binns y por Eberhart \& Russell son $13,4,3$ y 11 , y los genotipos 9,4 y 11 son los que podrían incorporarse según la ecovalencia de Wricke.

\section{Número de semillas}

En la Tabla 2 se observan los valores de ecovalencia de Wricke y del índice de Lin \& Binns. Los cinco genotipos que presentaron los valores más bajos de ecovalencia de Wricke fueron 13, 9 , 2, 5 y 12, considerados los más estables, pero 9 y 2 tienen una media inferior a la media general por lo cual no serán seleccionados. Los genotipos que tuvieron un valor más bajo de índice de Lin \& Binns fueron 10, 8, 13, 5 y 12, y todos tuvieron un rendimiento superior a la media general. Con respecto a los parámetros de Eberhart \& Russell, la prueba de hipótesis para $\beta_{1} \beta_{i}=1$ no hubo evidencia suficiente para rechazarla lo que indica adaptabilidad, general o pobre, según su media sea superior o inferior a la media general. Con respecto a $S_{d i}^{2}$, para los genotipos 4,8 y 11 resultó significativamente distinto de 0 , lo que indica que son inestables. Según esta metodología, se podrían seleccionar los genotipos 10, 13, 5 y 12 que poseen adaptación general, son estables y su media supera a la media general. Los valores del coeficiente de determinación $\left(R^{2}\right)$ aparecen tabulados en la última columna de la Tabla 4. Sus valores fueron muy bajos, y muestran que entre $0 \mathrm{y}$ $25,33 \%$ de la variación en la productividad de los genotipos se debe a la respuesta lineal de éstos frente a los cambios ambientales.

Para el número de semillas por planta el índice de Lin \& Binns y el método de Eberhart \& Russell coinciden en seleccionar los genotipos 10, 13, 5 y 12. Por la ecovalencia de Wricke se podrían seleccionar los genotipos 13, 5 y 12 .

\section{Peso de 1000 semillas}

En la Tabla 2 se observan los valores de ecovalencia de Wricke y del índice de Lin \& Binns. Los cinco genotipos que presentaron los valores más bajos de ecovalencia de Wricke fueron 9, 8, 13,3 y 5 y son considerados los más estables, pero 8 y 3 tienen una media inferior a la media general por lo cual no fueron seleccionados. Los genotipos que tuvieron un valor más bajo para el índice de Lin \& Binns fueron 2, 5, 9, 1 y 12 y todos tuvieron un rendimiento superior a la media general. Con respecto a los parámetros de Eberhart \& Russell, en la prueba de hipótesis para $\beta_{1} \beta_{i}=1$ no hubo evidencia suficiente para rechazarla, lo que indica adaptabilidad, general o pobre, según su media sea superior o inferior a la media general. Con respecto a la prueba de hipótesis para $S_{d i}^{2}$, su valor para los genotipos 4 y 7 resultó significativamente distinto de 0 lo que

Tabla 4. Parámetros de adaptabilidad $\left(\beta_{i}\right)$, de estabilidad $\left(S_{d i}^{2}\right)$, media general $(\mu)$ y coeficiente de determinación $\left(R^{2}\right)$ para el número de semillas por planta en cebadilla criolla en tres años de estudio.

\begin{tabular}{ccccrlrl}
\hline Genotipo & Media & $\begin{array}{c}\mu= \\
156,69\end{array}$ & $\beta_{i}$ & \multicolumn{1}{c}{$S_{d i}^{2}$} & Interpretación & $R^{2}$ \\
\hline 1 & 141,06 & $<$ & 0,70 & 218,95 & Adaptación pobre a todos los ambientes. Estable. & 3,12 \\
2 & 122,13 & $<$ & 1,07 & 110,83 & Adaptación pobre a todos los ambientes. Estable. & 12,10 \\
3 & 135,65 & $<$ & 3,72 & 170,11 & Adaptación pobre a todos los ambientes. Estable. & 25,33 \\
4 & 124,39 & $<$ & 2,02 & 1615,48 & Adaptación pobre a todos los ambientes. Inestable. & 15,26 \\
5 & 161,40 & $>$ & 0,12 & 112,91 & Adaptación general a todos los ambientes. Estable. Genotipo ideal. & 0,00 \\
6 & 152,49 & $<$ & 1,65 & 187,53 & Adaptación pobre a todos los ambientes. Estable. & 9,03 \\
7 & 151,89 & $<$ & 0,99 & 71,77 & Adaptación pobre a todos los ambientes. Estable. & 8,40 \\
8 & 202,57 & $>$ & 0,38 & 682,63 & Adaptación general a todos los ambientes. Inestable. & 2,32 \\
9 & 150,67 & $<$ & 0,20 & 246,92 & Adaptación pobre a todos los ambientes. Estable. & 0,00 \\
10 & 212,07 & $>$ & 0,77 & 287,44 & Adaptación general a todos los ambientes. Estable. Genotipo ideal. & 2,21 \\
11 & 124,48 & $<$ & 1,18 & 692,16 & Adaptación pobre a todos los ambientes. Inestable. & 8,45 \\
12 & 156,85 & $>$ & 2,29 & 26,56 & Adaptación general a todos los ambientes. Estable. Genotipo ideal. \\
13 & 188,26 & $>$ & 2,36 & 158,68 & Adaptación general a todos los ambientes. Estable. Genotipo ideal & 18,81 \\
13,21 \\
\hline
\end{tabular}


indica que son inestables. Según esta metodología, se podrían seleccionar los genotipos 2, 5, 9, 1 y 12 que poseen adaptación general, son estables y su media supera a la media general. Los valores del coeficiente de determinación $\left(R^{2}\right)$ aparecen tabulados en la última columna de la Tabla 5. Sus valores fueron variables ya que entre un $17,41 \mathrm{y}$ un $76,47 \%$ de la variación en la productividad de los genotipos se debe a la respuesta lineal de éstos frente a los cambios ambientales. Entre el índice de Lin \& Binns y el parámetro de estabilidad de Eberhart \& Russell hay total coincidencia en destacar los genotipos 2, 5, 9, 1 y 12. En cambio, con la ecovalencia de Wricke la coincidencia es sólo para los genotipos 5 y 9 .

Todos los procedimientos utilizados en este estudio se incluyen dentro del enfoque paramétrico, y como se puede apreciar es difícil llegar a una conclusión única con los 13 genotipos y las 3 variables analizadas. Lin et al. (1986) mencionan que la razón básica de esta dificultad es porque la respuesta de los genotipos a los ambientes es multivariada, aun cuando el enfoque paramétrico trata de transformarlo a un problema de una sola variable.

La metodología más usada en este tipo de estudios es la propuesta por Eberhart \& Russell. Sin embargo, Cruz y Salazar (1992) señalan que este procedimiento generó controversias por la supuesta violación de los supuestos de la regresión al utilizar como variable independiente los promedios por ambiente de la variable de respuesta. Las aproximaciones basadas en la técnica de regresión lineal son cuestionadas porque el índice ambiental, que habitualmente se usa como variable regresora, no es independiente de los datos analizados y los coeficientes de regresión pueden ser sesgados porque la variable independiente no es medida sin error. Revisando las consideraciones estadísticas, Skrøppa (1984) estableció que, con gran número de genotipos, ambientes y repeticiones, sin los outliers de cada genotipo o de los efectos ambientales y con las varianzas de los errores de los genotipos homogéneos, como usualmente es asumido en el ANOVA, la aproximación a la regresión podría ser estadísticamente válida.

Scapim et al. (2000) sostiene que la metodología de Lin \& Binns puede ser una buena alternativa para evaluar el rendimiento de un cultivar en la presencia de interacción genotipo-ambiente. La definición de superioridad es similar a las definidas en los objetivos de un programa de mejoramiento, ya que un cultivar superior debería estar entre los más productivos en el mayor número posible de ambientes (Farias et al., 1997; Scapim et al., 2000).

Considerando que la metodología de Lin \& Binns es fácil de aplicar e interpretar, siempre asocia mayor producción con mayor estabilidad y no presenta las restricciones de uso de la regresión, su uso resulta conveniente.

\section{AGRADECIMIENTOS}

Los autores agradecen el financiamiento recibido del Departamento de Ciencias Básicas de la Universidad Nacional de Luján para la realización de este trabajo.

\section{BIBLIOGRAFÍA}

Allard, R. W., 1960. Principles of Plant Breeding. John

Tabla 5. Parámetros de adaptabilidad $\left(\beta_{i}\right)$, de estabilidad $\left(S_{d i}^{2}\right)$, media general $(\mu)$ y coeficiente de determinación $\left(R^{2}\right)$ para el peso de 1000 de semillas en cebadilla criolla en tres años de estudio.

\begin{tabular}{|c|c|c|c|c|c|c|}
\hline Genotipo & Media & $\mu=9,55$ & $\beta_{i}$ & $S_{d i}^{2}$ & Interpretación & $R^{2}$ \\
\hline 1 & 10,19 & $>$ & 0,51 & 0,18 & Adaptación general a todos los ambientes. Estable. Genotipo ideal. & 17,41 \\
\hline 2 & 12,11 & $>$ & 1,89 & 0,18 & Adaptación general a todos los ambientes. Estable. Genotipo ideal. & 76,47 \\
\hline 3 & 8,12 & $<$ & 1,11 & 0,13 & Adaptación pobre a todos los ambientes. Estable. & 49,24 \\
\hline 4 & 8,56 & $<$ & 1,05 & 1,23 & Adaptación pobre a todos los ambientes. Inestable. & 41,10 \\
\hline 5 & 11,13 & $>$ & 0,86 & 0,07 & Adaptación general a todos los ambientes. Estable. Genotipo ideal. & 30,41 \\
\hline 6 & 8,05 & $<$ & 0,72 & 0,17 & Adaptación pobre a todos los ambientes. Estable. & 19,12 \\
\hline 7 & 8,75 & $<$ & 1,60 & 1,61 & Adaptación pobre a todos los ambientes. Inestable. & 67,33 \\
\hline 8 & 8,77 & $<$ & 0,92 & 0,13 & Adaptación pobre a todos los ambientes. Estable. & 74,30 \\
\hline 9 & 10,85 & $>$ & 1,08 & 0,09 & Adaptación general a todos los ambientes. Estable. Genotipo ideal. & 40,45 \\
\hline 10 & 8,87 & $<$ & 0,63 & 0,13 & Adaptación pobre a todos los ambientes. Estable. & 42,22 \\
\hline 11 & 9,06 & $<$ & 1,18 & 0,14 & Adaptación pobre a todos los ambientes. Estable. & 67,81 \\
\hline 12 & 10,06 & $>$ & 0,67 & 0,12 & Adaptación general a todos los ambientes. Estable. Genotipo ideal. & 40,41 \\
\hline 13 & 9,59 & $>$ & 0,77 & 0,10 & Adaptación general a todos los ambientes. Estable. Genotipo ideal. & 39,10 \\
\hline
\end{tabular}


Wiley \& Sons London, 485 pp.

Allard, R.W. and A.D. Bradshaw, 1964. Implications of genotype-environmental interactions in applied plant breeding. Crop Science 4:503-508.

Becker, H.C., 1981. Correlations among some statistical measures of phenotypic stability. Euphytica 30:835840.

Borém, A., 2001. Interação genótipo x ambiente, adaptabilidade e estabilidade de comportamento. In: Melhoramento de Plantas. 3. ed. Viçosa, Editora UFV, pp. 109-135.

Borém, A. y G.V. Miranda, 2009. Melhoramiento de plantas. Viçosa, Editora UFV. 529 pp

Campbell, B.T. and M.A. Jones, 2005. Assessment of genotype $\mathrm{x}$ environment interactions for yield and fiber quality in cotton performance trials. Euphytica 144:6978.

Chaves, L.J., 2001. Interacão de genótipos com ambientes. In: Recursos Genéticos e Melhoramento de plantas. (Eds). Nass, L.L., A.C.C. Valois, I.S. Melo, M.C. Valadares-Inglis. Fundação MT, Rondonópolis, pp. 673-713.

Cruz, M.R. y G.M. Salazar, 1992. Métodos alternativos en el análisis de la interacción genotipo-ambiente. Memorias Simposio Interacción Genotipo-Ambiente en genotecnia vegetal. Publicado por SOMEFI, Guadalajara, México, pp. 127-148.

Cruz, C.D.; A.D. Regazzi e P.C.S. Carneiro, 2004. Modelos biométricos aplicados ao melhoramento genético. Universidade Federal de Viçosa, Viçosa, Brasil, 480 pp.

Eberhart, S.A. and W.A. Russell, 1966. Stability parameters for comparing varieties. Crop sciences 6:36-40.

Elias, H.T.; S. Hemp, C.A. Scapim, M.A. Rodovalho, M.R. Royer, F. Mora and R.R. Barreto, 2005. Stability analysis of common bean genotypes in Santa Catarina State. Acta Scientiarum Agronomy 27:623-628.

Fan, X.M.; M.S. Kang, H. Chen, Y. Zhang, J. Tan and C. $\mathrm{Xu}, 2007$. Yield stability of maize hybrids evaluated in multi-environment trials in Yunnan, China. Agronomy Journal 99:220-228.

Farias, F.J.C.; M.A.P. Ramalho, L.R Carvalho, J.A.N. Moreira e J.N. Costa, 1997. Parámetros de estabilidade propostos por Lin e Binns (1988) comparados com - método da regressáo. Pesquisa Agropecuaria Brasileira 32:407-414.

Ferreira, D.F.; C.G.B. Demetrio, B.F.J. Manly, A.A. Machado and R. Vencovsky, 2006. Statistical models in agriculture: biometrical methods for evaluating phenotypic stability in plant breeding. Cerne 12:373-388.

Gauch, H.G. Jr., 1990. Full and reduced models for yield trials. Theoretical and Applied Genetcs. 80: 153-160.

Gauch Junior, H.G. and R.W. Zobel, 1996. AMMI analysis of yield trails. In: Kang, M.S.; Gauch Junior, H.G. (Ed.). Genotype by environment interaction. Boca Raton: CRC Press, pp.85-122.

InfoStat 2008. InfoStat version 2008. Grupo InfoStat. FCA, Universidad Nacional de Córdoba, Argentina.

Léon, J., 1986. Methods of simultaneous estimation of yield and yield stability. In: Biometrics in Plant Breeding. Proc. $6^{\text {th }}$ Meeting. Eucarpia, section Biometrics in Plant Breeding, Birmingham, pp. 299-308.

Léon, H.C. and C. Becker, 1988. Repeatability of some statistical measures of phenotypic stability correlations between single year results and multi years results. Plant Breeding 100:137-142.

Lin, C.S.; M.R. Binns and L.P. Lefkovitch, 1986. Stability analysis: where do we stand. Crop Science 26:894900.

Lin, C.S. and M.R. Binns, 1988. A superiority measure of cultivar performance for cultivar $\times$ location data. Canadian Journal of Plant Science 68:193-198.

Pandey, S. y J.E. Vargas, 1985. La interacción fenotipo medio-ambiente y su importancia en el mejoramiento intrapoblacional en plantas cultivadas. Actas VII Congreso Latinoamericano de Genética. I Congreso Colombiano de Genética. p. 38

Pereira, H.S.; L.C. Melo, M.J. del Peloso, L.C. Farias, L.G.C. da Costa, J.L.C. Diaz, C.A. Rava e A. Wendland, 2009. Comparação do método de análise de adaptabilidade e estabilidade fenotípica em feijoeiro-comum. Pesquisa Agropecuaria Brasileira 44:374-383.

Scapim, C.A.; V.R. Oliveira, A.L. Braccini, C.D. Cruz, C.A.B. Andrade and M.C.G. Vidigal, 2000. Yield stability in maize (Zea mays L.) and correlations among the parameters of the Eberhart and Russell, Lin and Binns and Huehn models. Genetics and Molecular Biology 23:387-393.

Skrøppa, T., 1984. A critical evaluation of methods available to estimate the genotype $x$ environment interaction. Studia Forrestalia Suecica 166:3-14.

Wolff, R.; L. Abbott and S. Pistorale, 1996. Reproductive behaviour of Bromus catharticus Vahl. (cebadilla criolla) in natural and cultivated populations. Journal of Genetics and Breeding 50:121-128.

Wolff, R.; L. Abbott and S. Pistorale, 2006. Estimation of genetic parameters in Bromus catharticus Vahl. Journal of Basic and Applied Genetics 17:51-59.

Wricke, G., 1962. Über eine Methode zur Erfassung der ökologischen Streubreite in Feldversuchen. Zeitschrift für Pflanzenzüchtung 47:92-96. 